\title{
Utilidad de mediciones objetivas para la programación en implantes cocleares
}

\author{
Use of objective measures for cochlear implant programming
}

Sofia Waissbluth A', Álvaro Del Valle $L^{2}$, César Toro A ${ }^{2,3}$.

\begin{abstract}
RESUMEN
Introducción: La programación adecuada de los implantes cocleares permiten lograr niveles de estimulación auditivos óptimos. Se realiza de forma individualizada, siendo la detección del umbral de confort ideal un desafío. Se ha descrito la utilidad de los potenciales auditivos del tronco encefálico eléctrico (ePEATC) y el reflejo eléctrico estapedial (eREE) para este propósito.

Objetivo: Determinar la posibilidad de realizar ePEATC y eREE en pacientes adultos y pediátricos con implantes cocleares, y evaluar cambios en la programación de los implantes cocleares luego de las mediciones objetivas.

Material y método: Se realizó un estudio prospectivo, descriptivo, de pacientes con implante coclear marca MED-EL ${ }^{\circledR}$, separando los pacientes en dos grupos: el grupo adulto $(n=5)$ y el grupo pediátrico $(n=5)$. Todos los pacientes incluidos presentaron más de 6 meses de encendido del implante. Se evaluaron variables epidemiológicas y tiempo de encendido del implante. Se realizó una otoscopía, prueba básica de funcionamiento del implante, y las mediciones objetivas eléctricas (ePEATC, eREE). Con estos resultados se ajustó el umbral de confort.

Resultados: El tiempo promedio de encendido del implante en el grupo adulto fue de 27 meses, y 30 meses en el grupo pediátrico. El ePEATC requiere más tiempo y cooperación al compararlo con eREE. Luego de estas evaluaciones objetivas, fue necesario el ajuste del umbral de confort en tres pacientes adultos, y en dos pacientes pediátricos. Fue necesario realizar una audiometría de campo libre para estimar el umbral de confort en dos pacientes pediátricos que no presentaron respuesta en eREE.

Conclusión: Fue posible realizar estas mediciones objetivas en pacientes adultos y pediátricos, siendo mejor tolerado y requiriendo menos tiempo, el eREE.
\end{abstract}

Palabras clave: Implante coclear, umbral de confort, mediciones objetivas.

\footnotetext{
1 Departamento de Otorrinolaringología, Pontificia Universidad Católica de Chile, Santiago, Chile.

2 Departamento de Otorrinolaringología, Complejo Asistencial Dr. Sótero del Río, Santiago, Chile.

3 Servicio de Otorrinolaringología, Clínica Universidad de los Andes, Santiago, Chile.
}

Los autores declaran no tener conflictos de interés.

Recibido el 11 de octubre, 2018. Aceptado el 25 de mayo, 2019. 


\section{ABSTRACT}

Introduction: Mapping a cochlear implant allows for adjusting ideal electrical stimulation limits. It is an individualized process and detecting the most comfortable loudness level can be challenging. The use of electrically evoked auditory brainstem response (ePEATC) and electrically evoked stapedius reflex thresholds (eREE) have been considered for this purpose.

Aim: To determine the feasibility of performing ePEATC and eREE on adult and pediatric patients with a cochlear implant, and to evaluate changes in programming following these objective measures.

Material and method: A prospective, descriptive study was completed, of patients with MED-EL ${ }^{\circledR}$ cochlear implants, separating patients into two groups: adults $(n=5)$ and children $(n=5)$. All of the patients included had their implants activated for 6 months or longer. Epidemiological variables and duration of implant activation were evaluated. Otoscopy, a basic implant functioning evaluation, and objective measures (ePEATC, eREE) were performed. With these results, comfortable loudness levels were adjusted.

Results: Average duration of implant activation was 27 months and 30 months, for adults and children respectively. Performing ePEATC required more time and cooperation as compared to eREE. Following the objective measures, adjustment of the comfortable loudness levels was required for three adult and two pediatric patients. Sound field audiometry was necessary for two pediatric patients in order to estimate the comfortable loudness levels because the eREE responses were absent.

Conclusions: It is feasible to perform these objective measures for both adult and pediatric patients, with eREE requiring less time and being better tolerated by patients.

Key words: Cochlear implant, most comfortable loudness level, objective measures.

\section{INTRODUCCIÓN}

El implante coclear representa la primera tecnología capaz de sustituir la función del órgano sensorial de la audición. Tiene, por el momento, como indicación principal la hipoacusia neurosensorial severa a profunda bilateral ${ }^{1}$. A contar de este año, el implante coclear será parte de la ley Ricarte Soto, la cual provee un implante coclear unilateral para los pacientes con hipoacusia neurosensorial severa a profunda $(70 \mathrm{~dB}-90 \mathrm{~dB})$ poslocutiva². En Chile, según el segundo estudio nacional de la discapacidad realizado por el Servicio Nacional de la Discapacidad (SENADIS), 8,2\% de los adultos y $2,2 \%$ de los niños y adolescentes, presentan una situación de discapacidad debido a la hipoacusia ${ }^{3}$. Es decir, existe una cantidad importante de personas, que, a pesar de ocupar audífonos, no logran un buen nivel de audición y podrían ser candidatos a un implante coclear. Por lo tanto, es importante que los profesionales relacionados con implantes cocleares tengan algunos conceptos en relación a su programación.

El implante es un transductor que transforma las señales acústicas en señales eléctricas que luego estimulan el nervio auditivo. La programación del implante es personalizada, pero en la práctica ésta sigue siendo limitada, debido a las dificultades para comprender y diagnosticar la integración de la señal a nivel individual ${ }^{4}$. Es importante programar adecuadamente el implante para lograr el mejor rango auditivo posible en cada paciente, con este objetivo se realizan una serie de pruebas. Por ejemplo, la telemetría de impedancia eléctrica nos asegura que los electrodos están funcionando; la telemetría de respuesta neural nos asegura que se estimuló eléctricamente el nervio auditivo, y también se ocupan pruebas de percepción del habla a través de encuestas y entrevistas ${ }^{5}$. Ninguna de estas pruebas nos permite ajustar los parámetros ideales para programar un implante.

La programación del implante coclear incluye especificar el mapa auditivo, determinar el umbral 
de estimulación eléctrica y el umbral de confort. El umbral de estimulación eléctrica se refiere a la menor cantidad de estimulación eléctrica que es percibida por el usuario del implante, mientras que el umbral de confort es considerado como la mayor cantidad de estimulación eléctrica para la cual el paciente percibe los sonidos sin que le incomode 4 . Para los pacientes pediátricos de menor edad (i.e. Ienguaje limitado, experiencia inadecuada con el sonido y no pueden dar respuestas conductuales confiables), y los pacientes con dificultades para proporcionar una retroalimentación adecuada, la programación del implante puede ser más dificultosa ${ }^{6}$. Una buena programación del implante es crucial y se realiza de forma individualizada, esto permite que el paciente implantado pueda:

- Detectar y discriminar los sonidos de la voz humana en relación al lenguaje oral,

- lograr niveles de audición óptimos,

- evitar experiencias desagradables que propicien el rechazo del implante, y

- evitar una percepción acústica alterada que impacte el desarrollo del lenguaje oral?

Existen algunas mediciones objetivas que se pueden realizar a través de los electrodos del mismo implante coclear como son los potenciales auditivos del tronco encefálico eléctrico (ePEATC) ${ }^{8}$ y el reflejo eléctrico estapedial (eREE) ${ }^{9,10}$. Se ha descrito que estas mediciones objetivas permiten evaluar la funcionalidad del implante, determinar si existe función en la vía auditiva hasta el tronco cerebral, y posiblemente ajustar el mapa auditivo ${ }^{8-10}$.

\section{OBJETIVO}

Determinar la posibilidad de realizar el ePEATC y el eREE en los pacientes adultos y pediátricos con implantes cocleares, y evaluar cambios en la programación de los implantes cocleares luego de las mediciones objetivas.

\section{MATERIAL Y MÉTODO}

\section{Pacientes}

Se realizó un estudio prospectivo, descriptivo, de todos pacientes con implante coclear de marca
MED-EL ${ }^{\circledR}$. El estudio cuenta con la aprobación del Comité de Ética del Servicio de Salud Metropolitano Sur Oriente. Todos los pacientes adultos, y padres de los pacientes pediátricos, firmaron un consentimiento informado para participar en el estudio. Todos los pacientes incluidos presentan hipoacusia neurosensorial bilateral severa-profunda con un implante coclear unilateral con más de 6 meses de encendido, y con un oído contralateral con anatomía del oído medio conservada y sin componente conductivo de hipoacusia. Este último punto es necesario para poder realizar el eREE.

De un total de 64 pacientes implantados en el complejo asistencial Dr. Sótero del Río, 18 son pacientes adultos y 46 son pacientes pediátricos. En el grupo de adultos, 8/18 tiene un implante de marca MED-EL ${ }^{\circledR}$. Se excluyeron de este trabajo 3 pacientes: 2 por no presentarse al estudio, a pesar de aceptar la participación (un paciente de regiones), y uno por tener menos de 6 meses de encendido del implante. En el grupo pediátrico, se eligieron los pacientes que presentaron alguna dificultad para la programación de su implante $(n=5)$ : un paciente con neuropatía auditiva, un paciente con cavidad común, dos pacientes con bajo umbral de tolerancia y un paciente con sobreestimación del umbral (Figura 1).

Se eligieron los pacientes con implante de marca MED-EL ${ }^{\circledR}$ para establecer grupos similares, con un mismo dispositivo que presenta las mismas escalas de cargas, y mismo programa computacional de calibración para la conexión con el equipo de potenciales evocados.

\section{Evaluaciones}

A todos los pacientes $(n=10)$, se les realizó una otoscopía para evaluar la indemnidad de las estructuras anatómicas del conducto auditivo y ausencia de cerumen o de efusión. Se revisaron los resultados de la última telemetría de respuesta neural disponible para cada paciente, para tener seguridad que el implante coclear esté en buenas condiciones funcionales y comprobar la integridad de la conducción de la energía eléctrica a través de los electrodos. Posteriormente, se realizaron las mediciones objetivas eléctricas: eREE y ePEATC.

Para la obtener el eREE, se utilizó el impedanciómetro de marca GSI modelo Tympstar, junto con la interface $[\mathrm{DIB}=$ Diagnostic Interface Box] de implante 


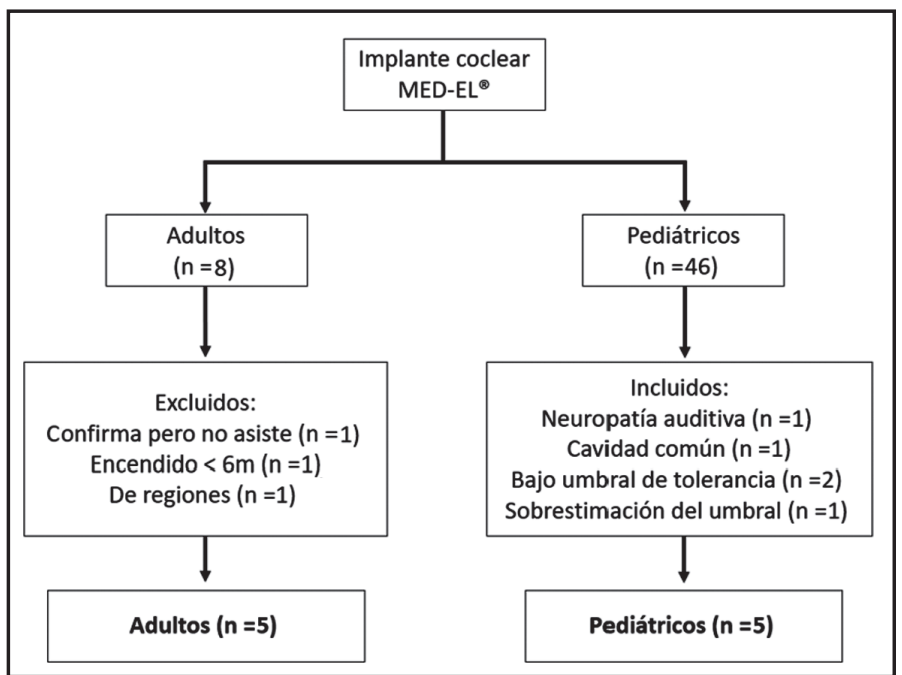

Figura 1. Reclutamiento de los pacientes adultos y pediátricos con implante coclear marca MED-EL ${ }^{\circledR}$.

coclear de la compañía MED-EL ${ }^{\circledR}$. En el oído implantado conectamos la bobina de la interface al paciente, y en el oído contralateral se introduce la sonda del impedanciómetro. Se realiza la estimulación eléctrica (tono burst de 500 milisegundos) a través de la interface del implante para capturar la actividad de la vía eferente contralateral del reflejo estapedial en el oído no implantado. Para esto, se utiliza la ventana de deterioro de reflejo (ventana de 15 segundos) con estímulo externo. Se registra el reflejo a través de la pantalla de análisis del tone decay dado que presenta una duración de registro de 15 segundos. Se mide el reflejo en 5 electrodos; uno basal, uno apical y 3 medios, correspondientes a los números 1, 5, 811 y 12 (e1, e5, e8, e11 y e12) en cada paciente, iniciando las mediciones en los electrodos apicales y luego hacia los basales, comenzando por un estímulo de baja carga hasta obtener el umbral. La intensidad de carga eléctrica con la cual se desencadena el umbral del eREE se utilizó como máximo umbral de confort para la programación de los implantes. El criterio para la determinación del umbral fue por inspección visual. En la Figura 2, podemos observar un ejemplo de registro para un paciente adulto.

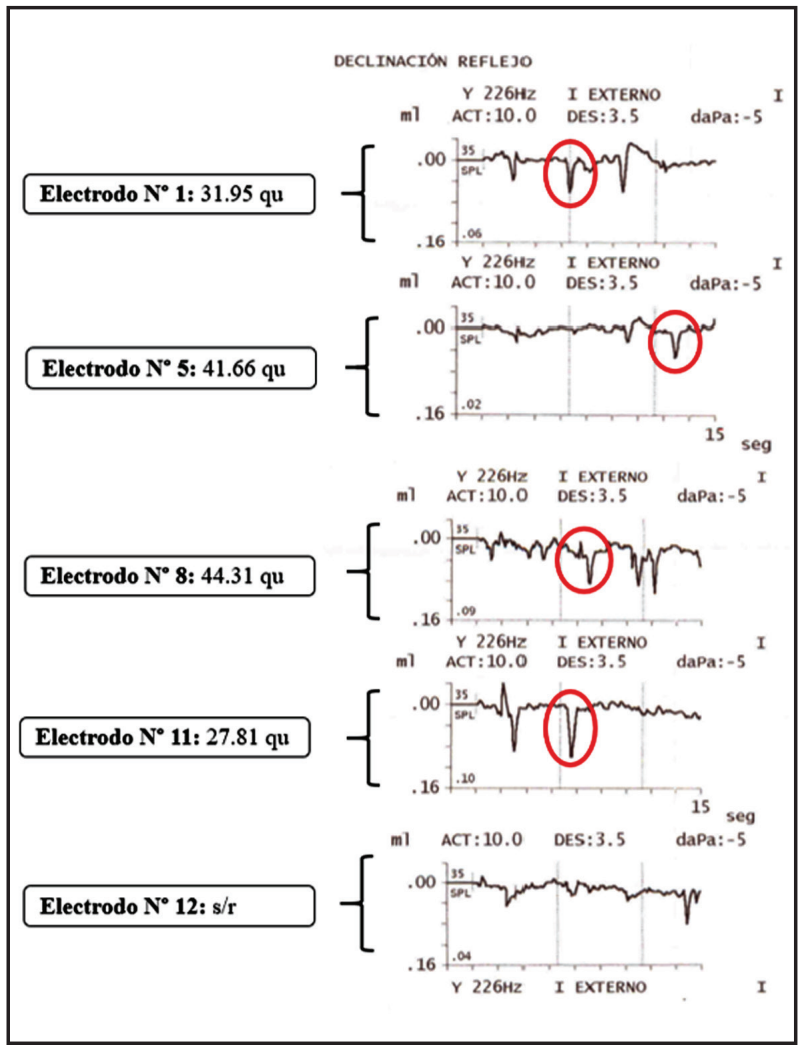

Figura 2. Registro de un eREE para un paciente adulto. Registro de 5 electrodos para un paciente adulto (paciente \#2). Se destaca umbral del reflejo en rojo. El reflejo se observa como una espiga invertida, y no como una meseta, debido a que la duración del burst es muy pequeña. 
Para el registro de ePEATC, se utilizó el equipo Eclipse EP25 de la marca Interacoustics ${ }^{\circledR}$ junto con la interface [DIB] de implante coclear de la compañía MED-EL ${ }^{\circledR}$. Se utilizó el programa de computación maestro 6.0. Se limpia la piel del paciente con Nuprep ${ }^{\circledR}$ (o algún tipo de gel abrasivo) en la zona de la frente y mastoides contralateral al implante, y se colocan los electrodos desechables. Luego, se comprueba la impedancia $(0<0 \mathrm{~K}<5$ $\mathrm{K} \Omega$ ). En el oído implantado, conectamos la bobina para realizar la estimulación, y la captura del registro del EPEATC se realiza en forma contralateral a través de 3 electrodos (positivo, negativo y tierra). Se realizan 2.000 promediaciones para obtener en el registro por electrodo para determinar el umbral de registro de carga eléctrica con la cual se desencadena, se miden los mismos electrodos anteriormente mencionados. Los parámetros de medición son los siguientes: promediación 2.000/4 estímulos, amplitud de ventana de registro 0,5 - 1 $\mu \mathrm{V}$, filtro pasa banda $(30-3.000 \mathrm{~Hz})$, filtro notch 50 $060 \mathrm{~Hz}$ y tasa de estímulo $34 \mathrm{~Hz}$. El criterio para la determinación del umbral fue por inspección visual. En la Figura 3, podemos observar un ejemplo de registro para un paciente adulto.

\section{RESULTADOS}

El estudio incluye un total de 10 pacientes (Figura 1). El grupo adulto $(n=5)$ presenta una edad promedio de 44 años (rango: 22-61 años) y el grupo pediátrico $(n=5)$ presenta una edad promedio de 7,4 años (rango: de 5-11 años). El tiempo promedio de encendido del implante en el grupo adulto fue de 27 meses (rango: 9-41 meses), y de 30 meses en el grupo pediátrico (rango: 8-60 meses) (Tabla 1).

El ePEATC requirió más tiempo y cooperación por parte del paciente. El tiempo de evaluación de los 5 electrodos fue, aproximadamente, de 1 hora para el ePEATC y 20 minutos para el eREE. Fue posible realizar el ePEATC y eREE en todos los pacientes adultos. No fue posible obtener respuesta en el ePEATC en al menos un electrodo para 5 pacientes, principalmente el electrodo 12, el cual corresponde a la base de la cóclea, zona más ancha de la cóclea y donde puede quedar el implante más lateralizado (Tabla 2).

Para el eREE, no fue posible obtener respuesta en al menos un electrodo para 3 pacientes, principalmente electrodo 12 (Tabla 4). No se observó

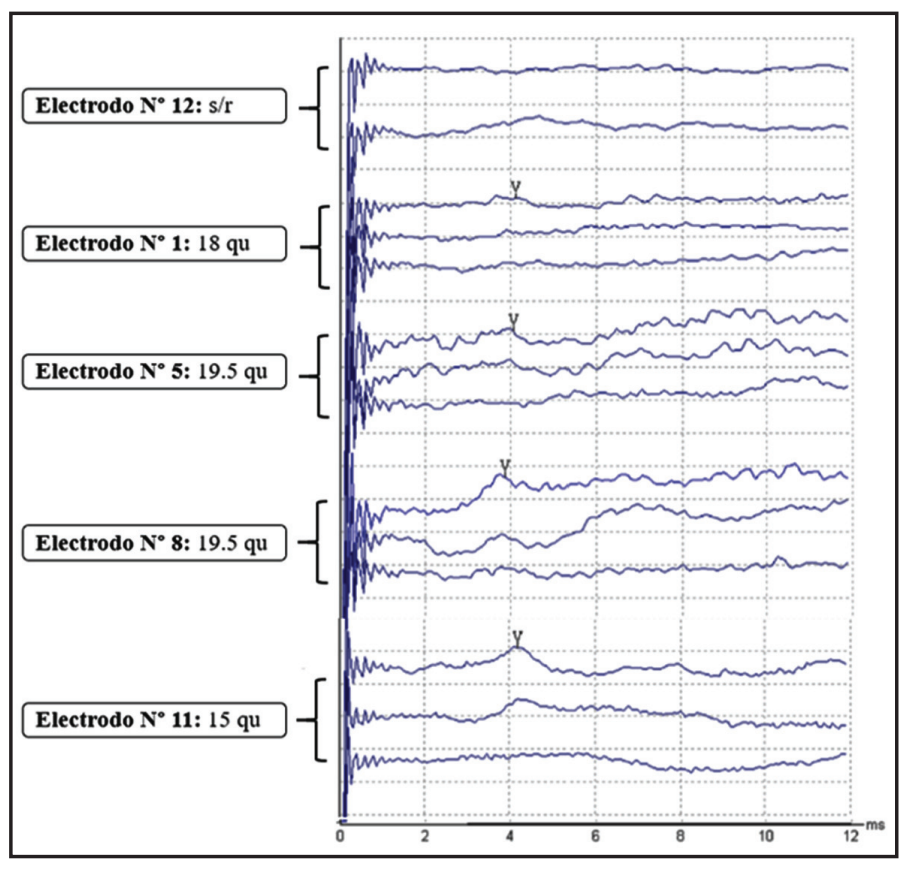

Figura 3. Registro de un ePEATC para un paciente adulto. Registro de 5 electrodos para un paciente adulto (paciente \#2) con umbral de onda $\mathrm{V}$ marcado. $\mathrm{s} / \mathrm{r}$ : sin respuesta. 
Tabla 1.

\begin{tabular}{|cccccl|}
\hline \multicolumn{5}{|c|}{ Pacientes adultos } \\
\hline Paciente & $\begin{array}{c}\text { Edad } \\
\text { (años) }\end{array}$ & $\begin{array}{c}\text { Encendido } \\
\text { (meses) }\end{array}$ & $\begin{array}{c}\text { PTP dB } \\
\text { preimplante }\end{array}$ & $\begin{array}{c}\text { PTP dB } \\
\text { posimplante* }\end{array}$ & Etiología \\
\hline 1 & 22 & 9 & 106.6 & 40 & HNS adquirida (posmeningitis) \\
2 & 33 & 34 & 81.3 & 37.5 & HNS progresiva poslingual, probable causa genética \\
3 & 50 & 41 & $\mathrm{~s} / \mathrm{r}$ a 110 & 46.25 & HNS progresiva poslingual, probable causa genética \\
4 & 55 & 19 & $\mathrm{~s} / \mathrm{r}$ a 110 & 37.25 & HNS progresiva poslingual, probable causa genética \\
5 & 61 & 31 & 103 & 33.75 & HNS adquirida (posmeningitis) \\
\hline & & & & Pacientes pediátricos & \\
\hline 1 & 9 & 37 & 83 & 40 & HNS congénita progresiva bilateral \\
2 & 6 & 29 & $\mathrm{~s} / \mathrm{r}$ a 110 & 37.5 & Síndrome de Waardenburg \\
3 & 6 & 18 & $\mathrm{~s} / \mathrm{ra} 90$ & 46.25 & HNS congénita (malformación coclear: cavidad común) \\
4 & 11 & 8 & 67,5 & 37.25 & Desorden en el espectro de la neuropatía auditiva \\
5 & 5 & 60 & $\mathrm{~s} / \mathrm{r}$ a 110 & 33.75 & HNS congénita profunda prelingual \\
\hline
\end{tabular}

s/r: sin respuesta, PTP: cálculo incluye frecuencias $0.5,1,2$ y $4 \mathrm{kHz}$ con audiometría de tonos puros (preimplante; oído a implantar) y de campo libre (posimplante). ${ }^{*}$ En promedio, a los dos años de la cirugía de implante coclear. Si el paciente no ha presentado un tiempo posoperatorio $\geq 2$ años, el PTP corresponde al máximo tiempo de encendido del implante descrito. HNS: hipoacusia neurosensorial.

Tabla 2. Resultados de telemetría de respuesta neural y ePEATC para los pacientes adultos

\begin{tabular}{|c|c|c|c|c|c|}
\hline Paciente & \multicolumn{5}{|c|}{ Telemetría de respuesta neural } \\
\hline & e1 & e5 & e8 & e11 & $\mathrm{e} 12$ \\
\hline 1 & 1 & 1 & 1 & 1 & 1 \\
\hline 2 & 1 & 1 & 1 & 1 & 1 \\
\hline 3 & 1 & $N / E$ & $\mathrm{~N} / \mathrm{E}$ & $s / r$ & $s / r$ \\
\hline 4 & 1 & 1 & 1 & 1 & $s / r$ \\
\hline 5 & 1 & $s / r$ & 1 & $s / r$ & $s / r$ \\
\hline \multicolumn{6}{|c|}{ ePEATC } \\
\hline 1 & 21 & 19.5 & 21 & 19.5 & 25.5 \\
\hline 2 & 18 & 19.5 & 19.5 & 15 & $s / r$ \\
\hline 3 & 12 & 12 & 16.5 & $s / r$ & $s / r$ \\
\hline 4 & 15 & 18 & 18 & 19.5 & $s / r$ \\
\hline 5 & 15 & 15 & $s / r$ & 16.5 & $s / r$ \\
\hline
\end{tabular}

Telemetría: 1 =respuesta, N/E: no evaluado, $s / r=s i n$ respuesta. ePEATC $s / r=s i n$ respuesta evaluada hasta 36 unidades de carga, ePEATC: resultados en unidades de carga.

una relación entre tiempo de encendido o duración de encendido del implante con la falta de respuestas. Tampoco se puede establecer una relación con la duración de la hipoacusia ya que existe considerable variabilidad entre los pacientes para analizar esta variable. En el caso de paciente adulto \#1, observamos respuestas en todos los electrodos en todas las mediciones (ePEATC, eREE y telemetría). A partir de los resultados obtenidos por el eREE, se redujo la carga eléctrica de tres electrodos; e1, e8 y e11. Para el paciente adulto \#2, se obtuvo respuestas en todos los electrodos en la telemetría, pero no se obtuvo respuesta en el e12 en el ePEATC ni en el eREE. A partir de los resultados obtenidos por el eREE, se redujo la carga eléctrica de dos electrodos; e5 y e8. Para el paciente adulto \#4, observamos respuestas en todos los electrodos, excepto e12, para la telemetría, ePEATC y eREE. A partir de 
Ios resultados obtenidos por el eREE, se redujo la carga eléctrica de tres electrodos; e1, e5 y e8. Por lo tanto, se requirió un ajuste al umbral de confort en dos o más electrodos en 3 pacientes adultos, y esto fue posible gracias a los resultados de eREE. Si no se observó respuesta en un electrodo en particular, no se realizó cambio a ese electrodo. Hubo respuesta en $76 \%$ de los electrodos evaluados por ePEATC, y $88 \%$ por eREE. Para el grupo de pacientes pediátricos (Tabla 3), se eligieron a los pacientes que presentaron alguna dificultad para la programación del implante. En el caso de dos pacientes, fueron incluidos en el estudio ya que presentaron un bajo umbral de tolerancia, es decir, con poca carga, les resultaba molesto el uso del implante (pacientes pediátricos \#1 y \#5). No fue posible obtener respuesta en ningún electrodo evaluado durante el ePEATC para dos pacientes (pacientes pediátricos \#4 y \#5). No fue posible obtener respuesta en ningún electrodo evaluado durante el eREE para dos pacientes (pacientes pediátricos \#2 y \#3) (Tabla 4).

Para el paciente pediátrico \#1, observamos respuestas en todos los electrodos, en la telemetría, ePEATC y eREE. A partir de los resultados obtenidos por el eREE, se redujo la carga eléctrica de cuatro electrodos; e5, e8, e11 y e12. Para el paciente pediátrico \#5, se obtuvieron respuestas en la telemetría y el eREE, pero no con ePEATC. Gracias a los resultados de eREE, se redujo la carga eléctrica a tres electrodos: e5, e8 y e11. En el caso del paciente pediátrico \#2, fue incluida en el estudio por presentar una sobreestimación del umbral. Este paciente presenta un trastorno del espectro autista, y problemas conductuales, que dificultaron la calibración adecuada del implante de forma conductual. Se observaron respuestas en todos los electrodos, tanto en la telemetría como en el ePEATC, esto no fue así en el eREE. Debido a la falta de respuesta en el eREE, se requirió realizar audiometría de campo libre para obtener alguna idea del umbral de confort. Algo similar ocurrió con paciente pediátrico \#3, el cual presenta una cavidad común. Se obtuvo respuesta en la telemetría en el electrodo e8, y respuestas en el ePEATC en Ios electrodos e8 y e12, pero ninguna respuesta en eREE. En este caso, también fue necesario realizar una audiometría de campo libre para obtener alguna idea del umbral de confort. No se observó una relación entre tiempo de encendido o duración de encendido del implante con la falta de respuestas. Hubo respuesta en $44 \%$ de los electrodos evaluados por ePEATC, y $60 \%$ por eREE.

La reducción de la carga se realizó en los umbrales de confort, es importante considerar que eléctricamente el umbral de confort en un rango de carga que el paciente asocia a buena audibilidad sin molestia auditiva. Se consideró el valor del eREE como referencia para el umbral de confort por electrodo, y se realizó una reducción paulatina

Tabla 3. Resultados de telemetría de respuesta neural y ePEATC para los pacientes pediátricos

\begin{tabular}{|c|c|c|c|c|c|}
\hline \multirow[t]{2}{*}{ Paciente } & \multicolumn{5}{|c|}{ Telemetría de respuesta neural } \\
\hline & e1 & e5 & e8 & e11 & e12 \\
\hline 1 & 1 & 1 & 1 & 1 & 1 \\
\hline 2 & 1 & 1 & 1 & 1 & 1 \\
\hline 3 & $s / r$ & $s / r$ & 1 & $s / r$ & $s / r$ \\
\hline 4 & 1 & 1 & 1 & 1 & 1 \\
\hline 5 & 1 & 1 & 1 & 1 & 1 \\
\hline \multicolumn{6}{|c|}{ ePEATC } \\
\hline 1 & 16.68 & 20.01 & 23.04 & 20.01 & 20.01 \\
\hline 2 & 18 & $\mathrm{~s} / \mathrm{r}$ & 16.5 & 16.5 & 13.5 \\
\hline 3 & $s / r$ & $s / r$ & 22.5 & $\mathrm{~s} / \mathrm{r}$ & 21 \\
\hline 4 & $s / r$ & $s / r$ & $s / r$ & $s / r$ & $s / r$ \\
\hline 5 & $s / r$ & $s / r$ & $s / r$ & $s / r$ & $s / r$ \\
\hline
\end{tabular}

Telemetría: 1 =respuesta, $s / r=s i n$ respuesta. ePEATC $s / r=s i n$ respuesta evaluada hasta 36 unidades de carga, ePEATC: resultados en unidades de carga. Telemetría realizada previo al estudio. 
Tabla 4. Resultados de eREE para los pacientes adultos y pediátricos

\begin{tabular}{|c|c|c|c|c|c|c|c|c|c|c|c|}
\hline \multicolumn{7}{|c|}{ Pacientes adultos } & \multicolumn{5}{|c|}{ Pacientes pediátricos } \\
\hline & $\# 1$ & $\# 2$ & \#3 & $\# 4$ & $\# 5$ & & $\# 1$ & \#2 & $\# 3$ & $\# 4$ & $\# 5$ \\
\hline e1 pre & 40.47 & 37.67 & 15.77 & 33.67 & 24.4 & e1 pre & 21.21 & 86.68 & 63.33 & 21.97 & 42.76 \\
\hline e1 & 32.9 & 31.95 & 16.73 & 21.09 & 24.07 & e1 & 19.11 & $s / r$ & $s / r$ & 18.95 & 42.76 \\
\hline$\Delta$ & 7.57 & 5.72 & -0.96 & 12.58 & 0.33 & $\Delta$ & 2.1 & - & - & 3.02 & 0 \\
\hline e5 pre & 41.68 & 61.18 & 16.73 & 42.69 & 28.28 & e5 pre & 27.5 & 85.34 & 55.81 & 21.97 & 51 \\
\hline e5 & 38.14 & 41.66 & 18.83 & 24.35 & 27.46 & e5 & 19.87 & $s / r$ & $s / r$ & 20.11 & 42.71 \\
\hline$\Delta$ & 3.54 & 19.52 & -2.1 & 18.34 & 0.82 & $\Delta$ & 7.63 & - & - & 1.86 & 8.29 \\
\hline e8 pre & 44.22 & 59.54 & 19.4 & 51.49 & 31.78 & e8 pre & 31.08 & 78.28 & 54.28 & 21.97 & 53.11 \\
\hline e8 & 34.91 & 44.31 & 25.31 & 34.35 & 34.72 & e8 & 19.95 & $s / r$ & $s / r$ & 19.52 & 45.81 \\
\hline$\Delta$ & 9.31 & 15.23 & -5.91 & 17.14 & -2.94 & $\Delta$ & 11.13 & - & - & 2.45 & 7.3 \\
\hline e11 pre & 46.91 & 27.8 & 16.24 & 26.6 & 29.16 & e11 pre & 36.96 & 77.24 & 61.1 & 21.97 & 51.53 \\
\hline e11 & 38.14 & 27.8 & 34.01 & 25.83 & $s / r$ & e11 & 20.46 & $s / r$ & $s / r$ & 20.11 & 45.16 \\
\hline$\Delta$ & 8.77 & 0 & -17.77 & 0.77 & - & $\Delta$ & 16.5 & - & - & 1.86 & 6.37 \\
\hline e12 pre & 42.93 & 31.68 & 16.24 & 35.75 & 29.16 & e12 pre & 35.88 & 78.28 & 58.25 & 21.97 & 48.11 \\
\hline e12 & 40.47 & $s / r$ & 34.01 & $s / r$ & 24.16 & e12 & 22.36 & $s / r$ & $s / r$ & 36.31 & 42.3 \\
\hline$\Delta$ & 2.46 & - & -17.77 & - & 5 & $\Delta$ & 13.52 & - & - & -14.34 & 5.81 \\
\hline
\end{tabular}

Resultados descritos en unidad de carga (qu). Valores pre corresponden al valor por percepción del umbral de confort. $\Delta=$ diferencia en carga eléctrica post eREE.

de carga, con la intención de aproximar el umbral del eREE. Esto también controlado por su umbral tonal con implante, junto con pruebas de percepción auditiva.

\section{DISCUSIÓN}

La programación del implante coclear, o mapping, es un proceso individualizado que tiene como objetivo especificar un mapa auditivo, es decir poder establecer los niveles de estimulación eléctricos precisos para poder lograr un rango de audición ideal para el paciente implantado, siendo la determinación del umbral de confort un desafío ${ }^{4}$. El umbral de confort se puede estimar con medidas conductuales, aunque en niños pequeños 0 personas con dificultades para comunicarse 0 proporcionar un feedback adecuada, esto puede ser bastante complejo. Se ha descrito la utilidad de mediciones objetivas que se realizan a través de los electrodos del mismo implante coclear, como el ePEATC ${ }^{8}$, el eREE ${ }^{9,10}$, y la medición del potencial de acción eléctrico con el uso de la telemetría de la respuesta neural (ECAP: electrically evoked compound action potential) ${ }^{11}$.

Este último ha sido de utilidad para confirmar la funcionalidad del implante en el tiempo y para estimar el umbral de confort ${ }^{12}$. Sin embargo, los resultados obtenidos con el ECAP no se correlacionan necesariamente con las mediciones del eREE, y requiere de más tiempo para realizar el examen ${ }^{13}$. En pacientes implantados que presentan comorbilidades, existe una cooperación limitada lo que hace que la obtención de los valores del umbral de confort no pueda registrarse en forma adecuada. Se ha considerado el uso del eREE para determinar el umbral de confort en adultos 
y pacientes pediátricos ${ }^{14-16}$. Se logra realizar en la mayoría de los casos, aunque puede presentar un resultado negativo en casos de otitis media con efusión, membrana timpánica muy rígida o laxa, y también en algunos pacientes con oído medio sin alteraciones ${ }^{13,15,16}$. En algunos casos, no se puede encontrar una explicación por la cual no hay respuesta en el eREE. Kosaner y cols (2009) no obtuvieron respuesta en el eREE en 43 de 254 pacientes pediátricos evaluados; en 15 pacientes, no fue posible encontrar una razón por la cual no presentaron respuesta ${ }^{17}$. Este fue el caso igualmente con el estudio actual, para dos pacientes pediátricos; en ningún electrodo evaluado fue posible detectar eREE. En todos los pacientes adultos fue posible detectar eREE en más de un electrodo. Los valores de carga igualmente varían de forma importante según el paciente por lo cual la medición secuencial de eREE para un paciente es más lógico y útil que compararlo a otros pacientes implantados. Se ha observado que los niveles de eREE se estabilizan a los 4 a 6 meses de uso del implante ${ }^{17,18}$, y por esa razón, uno de los criterios de inclusión de este estudio fue el encendido del implante por más de 6 meses. Además, los estudios han demostrado una alta correlación entre el eREE y el nivel de confort conductual11,17.

El ePEATC también ha sido estudiado para la programación de los implantes cocleares. Su utilidad radica en que puede evaluar la integridad neural hasta el tronco cerebral. Sin embargo, la medición del ECAP, que es más rápida y no requiere sedación, nos entrega información similar sobre la respuesta del nervio auditivo a una un estímulo eléctrico. Por esta razón, el interés por la posible utilidad del ePEATC para la programación de los implantes cocleares ha disminuido ${ }^{19}$. Uno de los problemas principales en la realización del ePEATC en pacientes pediátricos es la cooperación ya que este examen requiere de más tiem$\mathrm{po}^{11}$. Aun con estas limitaciones, el ePEATC puede ser de gran utilidad en casos de mapas erróneos que han sido pasados por alto con mediciones de ECAP o del eREE ${ }^{6}$, en casos de disfunción/ disincronía de la vía auditiva ${ }^{11,20}$, o en casos en los cuales no fue posible detectar un ECAP ${ }^{19}$. Es decir, casos para los cuales la programación puede ser más dificultosa. En este estudio, no fue posible detectar una respuesta de ePEATC en dos pa- cientes pediátricos en ninguno de los electrodos evaluados. No tenemos una explicación clara para este fenómeno. Se debe tomar en consideración que los pacientes que fueron seleccionados para participar en este estudio presentaron dificultades en la programación inicialmente, y, por lo tanto, este hecho podría corresponder a un sesgo de selección.

El ePEATC confirma los resultados de la telemetría de respuesta neural, pero no es recomendable para la programación de los implantes. No presentó ventaja para programar el implante coclear mientras que el eREE sí permitió determinar el valor del umbral de confort en 8/10 pacientes. Esto se puede observar con la presencia y no presencia de respuesta, y además con la carga necesaria para evocar respuesta. Gracias al eREE, en el grupo adulto, tres pacientes requirieron un ajuste del umbral con una reducción del nivel de máxima comodidad, mientras que, en el grupo pediátrico, dos de los pacientes evaluados requirieron de esta reducción. Además, se acudió a una audiometría de campo libre para el ajuste del umbral de confort en dos pacientes pediátricos que no presentaron respuesta en el eREE.

El caso pediátrico de desorden en el espectro de la neuropatía auditiva (paciente pediátrico \#4), el paciente no presentó respuesta en el ePEATC, pero sí en el eREE. Fue difícil realizar la programación de su implante ya que no presentaba respuesta conductual adecuada, razón por lo cual se reclutó para este estudio. La respuesta del ePEATC es muy variable en estos pacientes. Shallop y col realizaron ePEATC a 4 niños implantados con desorden en el espectro de la neuropatía auditiva. Describen la presencia de respuesta en al menos un electrodo, pero no entregan información específica con respecto al procedimiento ${ }^{21}$. Por otro lado, Jeon y cols. observaron que 6 de 11 pacientes con desorden en el espectro de la neuropatía auditiva que fueron implantados, con seguimiento posimplante de 40,4 meses y con evaluación con ePEATC después de un año desde la cirugía, no presentaron respuesta ${ }^{22}$. Una posible explicación para la variabilidad de las respuestas a estas mediciones objetivas es el posible sitio originario de la disfunción. La ausencia de respuesta en el ePEATC del paciente 
pediátrico \#4 se puede deber a una disfunción postsináptica ${ }^{23}$. Se requieren estudios adicionales para dilucidar esta patología y sus características fisipatológicas.

Ambas mediciones, ePEATC y eREE, se pueden realizar en adultos y niños. El eREE es más confiable, y es bien tolerado por los pacientes pediátricos; no le molesta el estímulo a diferencia de lo que se ha descrito en la literatura con el ePEATC ${ }^{11}$. El eREE es una herramienta especialmente útil para pacientes con dificultad al entregar retroalimentación adecuada, y también para niños pequeños para los cuales puede ser muy difícil determinar el nivel de confort de forma conductual.

\section{BIBLIOGRAFÍA}

1. Egilmez, OK, Kalcioglu MT. Cochlear implant: indications, contraindications and complications. Scripta Scientifica Medica 2015; 47: 9-16.

2. Ministerio de Salud. Ley "Ricarte Soto" aumentará su cobertura a 18 patologías de alto costo. Disponible en: http://www.minsal. $\mathrm{cl} /$ ley-ricarte-soto-aumentara-su-cobertura-a18-patologias-de-alto-costo/. Consultado el 6 de junio 2018.

3. Servicio Nacional de la Discapacidad. II Estudio Nacional de la Discapacidad. Disponible en: https://www.senadis.gob.cl/pag/355/1197/ii estudio_nacional_de_discapacidad. Consultado el 6 de junio 2018.

4. Banda RI, Castillo S, Roque G. Parámetros de programación del implante coclear. Boletín médico del Hospital Infantil de México, 2017; 74: 65-9.

5. LENARZ T. Cochlear implant - state of the art. GMS Curr Top Otorhinolaryngol Head Neck Surg 2017; 16: Doc04.

6. Raghunandhan S, Ravikumar A, Kameswaran M, Mandke K, Ranjith R. Electrophysiological Correlates of Behavioral Comfort Levels in Cochlear Implantees: A Prospective Study. Indian J Otolaryngol Head Neck Surg 2015; 67: 210-22.

7. Shapiro WH, Bradham TS. Cochlear implant programming. Otolaryngol Clin North Am 2012; 45: 111-27.

\section{CONCLUSIÓN}

El ePEATC no fue una medida útil para la programación del implante coclear en este estudio ya que los cambios realizados a la programación fueron principalmente a partir de los resultados de eREE; una medida objetiva útil, que requiere menos tiempo y cooperación al compararlo con el ePEATC. El eREE es una herramienta de mucha utilidad para determinar el nivel de confort de forma no conductual. Como objetivo futuro, se realizará un estudio prospectivo que evalúe el beneficio de ajustar el umbral de confort con el eREE en la rehabilitación auditiva pediátrica.

8. Brown CJ, Hughes ML, Luk B, Abbas PJ, Wolaver A, GeRvals J. The relationship between EAP and EABR thresholds and levels used to program the nucleus 24 speech processor: data from adults. Ear Hear 2000; 21: 151-63.

9. Walkowiak A, Lorens A, Kostek B, Skarzynski H, Polak M. EREE, ART, and MCL correlations in experienced paediatric cochlear implant users. Cochlear Implants Int 2010; 11: 482-4.

10. Andrade KC, Leal M de C, Muniz LF, Menezes P de L, Albuquerque KM, Carnaúba AT. The importance of electrically evoked stapedial reflex in cochlear implant. Braz J Otorhinolaryngol 2014; 80: 68-77.

11. Gordon KA, Papsin BC, Harrison RV. Toward a battery of behavioral and objective measures to achieve optimal cochlear implant stimulation levels in children. Ear Hear 2004; 25: 447-63.

12. Carvalho B, Hamerschmidt R, Wiemes G. Intraoperative Neural Response Telemetry and Neural Recovery Function: a Comparative Study between Adults and Children. Int Arch Otorhinolaryngol 2015; 19: 10-5.

13. Kosaner J, Spitzer P, Bayguzina S, Gultekin M, Behar LA. Comparing eREE and eCAP measurements in pediatric MED-EL cochlear implant users. Cochlear Implants Int 2018; 19: 153-61.

14. SpIVAK LG, ChUTE PM. The relationship between electrical acoustic reflex thresholds and behavioral comfort levels in children and adult cochlear implant patients. Ear Hear 1994; 15: 184-92. 
15. Hodges AV, Balkany TJ, Ruth RA, Lambert PR, Dolan-Ash S, Schloffman JJ. Electrical middle ear muscle reflex: use in cochlear implant programming. Otolaryngol Head Neck Surg 1997; 117: 255-61.

16. Bresnihan M, Norman G, Scott F, Viani L. Measurement of comfort levels by means of electrical stapedial reflex in children. Arch Otolaryngol Head Neck Surg 2001; 127: 963-6.

17. Kosaner J, Anderson I, Turan Z, Deibl M. The Use of EREE in Fitting Children with Cochlear Implants. Int Adv Otol 2009; 5: 70-9.

18. Kosaner J. Generating Speech Processor Programmes for Children Using EREE Measurements. Cochlear implants international 2010; 11: 20-24.

19. Brown CJ. Clinical uses of electrically evoked auditory nerve and brainstem responses. Curr Opin Otolaryngol Head Neck Surg 2003; 11 : 383-7.
20. Mittal R,Ramesh AV, Panwar SS, Nilkanthan A, Sinha VR, NaIR S, RaJ P. Mapping of pediatric cochlear implant recipients using electrical auditory brainstem responses as a tool. Indian J Otol 2015; 21: 14-8.

21. Shallop JK, Peterson A, Facer GW, Fabry LB, DrIscoll CL. Cochlear implants in five cases of auditory neuropathy: postoperative findings and progress. Laryngoscope 2001; 111: 555-62.

22. Jeon JH, Bae MR, Song MH, Noh SH, Chol KH, CHо JY. Relationship between electrically evoked auditory brainstem response and auditory performance after cochlear implant in patients with auditory neuropathy spectrum disorder. Otol Neurotol 2013; 34: 1261-6.

23. McMahon CM, Patuzzi RB, Gibson WP, Sanli $H$. Frequency-specific electrocochleography indicates that presynaptic and postsynaptic mechanisms of auditory neuropathy exist. Ear Hear 2008; 29: 314-25. 